\title{
Wavelet Transform based Fusion Technique for Image Restoration
}

\author{
Kalpana Wagadre \\ M. Tech Scholar \\ ECE Department Radharaman Institute of \\ Technology and Science Bhopal, India
}

\author{
Madhu Singh \\ Assistant Professor \\ ECE Department Radharaman Institute of \\ Technology and Science Bhopal, India
}

\begin{abstract}
there is lots of image processing algorithms to improve image quality .Image restoration is a field of image processing which deals with restoring an image that has been degraded by some degradation phenomenon. Degradation may occur due to motion blur, Gaussian blur, noise or camera mismatch. The aim of image restoration is to reconstruct or estimate an uncorrupted image by using the degraded version of the same image. [2] In this paper we described a method to remove the motion blur present in the image taken from any cameras by which motion blurred and noisy image is first restored using Wiener and Lucy Richardson method then applied wavelet based fusion Technique is applied for restoration. The performance of the every stage is tabulated for the parameters like SNR and RMSE of the restored images. It is observed that image fusion technique provides better results as compared to previous techniques. Performance of all the methods has been compared on the basis of performance parameters MSE and PSNR.
\end{abstract}

\section{Keywords}

Image restoration, Image fusion, Wavelet, MSE, PSNR, Wiener, Lucy Richardson

\section{INTRODUCTION}

Images acquired through any image sensors consist of variety of noises (such as amplifier noise, , quantization noise and cross talk), resulting from stochastic variations and deterministic distortions or shading to extract fundamental image data from unrefined measured image data, some algorithms should be applied initially to ease noises and to pre-process images for further processing. For varying noise, and rapidly varying noise a linear and a nonlinear filter simuntanousaly can be applied for noise smoothing. Due to limited performance of both optical and electronic systems [8] Motion blur [9].occurs in various image development systems

Blur is usually modeled as a linear convolution of an image with a blurring kernel, also known as the point spread function (or PSF) [7].In remotely sensed images the relative motion is slow it results in fatal failure of detecting important information. Because of this reason, approach is to try to remove the blur off-line. The purpose of image restoration is to reconstruct or estimate an uncorrupted image by using the degraded version of the same image.

As blurring can degrade the visual quality of image with camera Photographers and low quality camera manufactures for example, The shutter speed is some time the main factor deciding the amount of motion blur. To reduces the degree of blur image could be capture using shorter exposure intervals in dark scenes increases the amount of noise in the image could increase there is many approach to try to remove the blur. Many image restoration methods such as iterative method. However, their computational complexity such as regularized iteration is so high that they can be implemented in real-time [1] .Shutter speed compare to object velocity, may degraded by motion blur. Blur can be modeled as a linear convolution of an input image with a blurring kernel, [3]. Relative motion is slow in remotely sensed images could results in fatal failure of detecting significant information [4].

By using restoration we can reconstruct or estimate an unaffected image. Linear motion blur with additive noise eq.1.1 shows the relationship between the observed image $\mathrm{g}(\mathrm{x}, \mathrm{y})$ and its pure version $\mathrm{f}(\mathrm{x}, \mathrm{y})[5]$.

$g(x, y)=f(x, y) * h(x, y)+v(x, y)$

The packages investigated for the development of the research application was MATLAB [9], [10,] which is commercially available and run on Microsoft Windows. The aim and objective of image restoration is to reconstruct or estimate an uncorrupted image by using the degraded version of the same image [5].

\subsection{Motion Blur}

Sometimes blur could be occurred in scene or picture due to Relative motion between camera and original scene atmospheric turbulence, out of focus of optical system and aberration in optical system medium some time also could introduce some noises in images that may cause of degradation, distortion or shading in original image So it become necessary to remove blur and reduce the amount of noise before image processing. Blur is a linear convolution of an original image with a blurring kernel, also known as the PSF.

\subsection{Wiener Filter}

Norbert Wiener first proposed the image restoration method in 1942. It is a non blind method in the method uses image and noise as random processes and use to find an estimate the original image. It performs the deconvolution by inverse filtering (high pass filter) and removes the noise with a compression operation (low pass filter) [15].

\subsection{Lucy Richardson Algorithm}

The Lucy Richardson (LR) algorithm is an iterative nonlinear restoration method that depends on the size and complexity of the PSF matrix. Small PSF sometime cause very smooth image and increasing iteration decelerate process and also produce ringing effect for the "good" quality of reconstructed image, the best possible no. of iterations are decided as per the PSF size [14].

\section{PROPOSED METHOD}

Restoration techniques used in our work are basically mathematical modeling of degradation of image and then 
applying inverse process in image to restore the original image. by removing noise present in image. In proposed method we compare different filter such as Wiener filter, Lucy Richardson and wavelet based image fusion technique for image restoration can be used for removal of motion blur present in affected image. Images restored are further compared on the basis of performance parameters such as PSNR and MSE [7].

\subsection{Image Restoration}

Image Restoration is to reconstruct the original image with a priori knowledge of the image degradation due to noise.

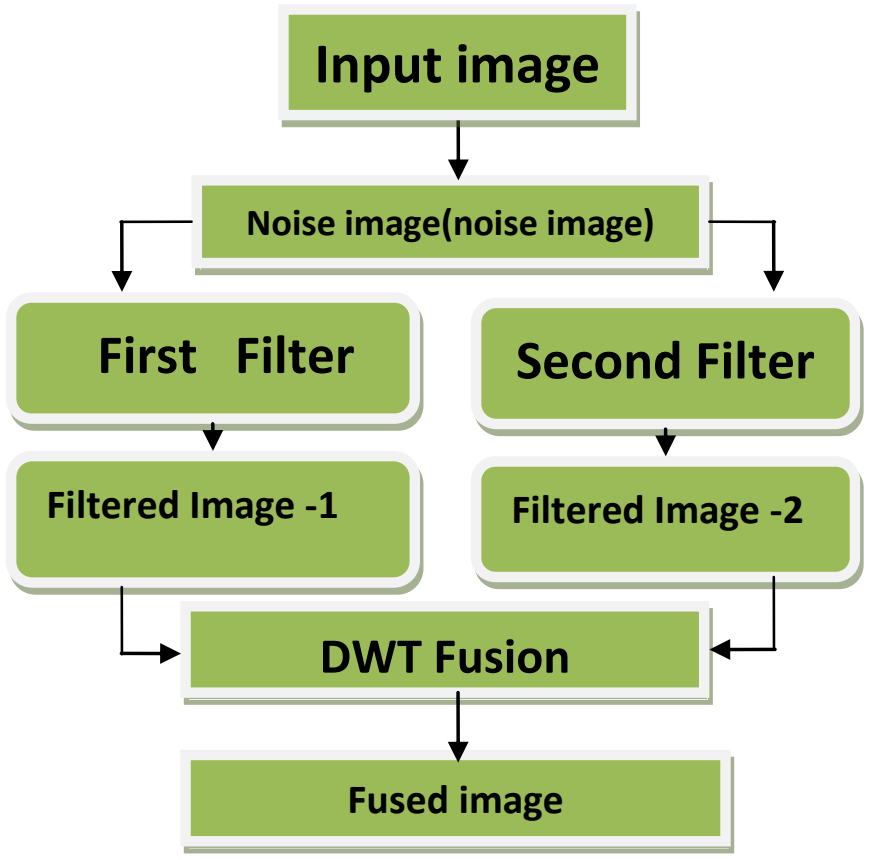

Fig 1 : Block diagram of proposed work

Noisy image is added with noise and then send to restoration filter which suppress the noise available in image and the output which is near to the original image. To remove blur of image Wiener and LR method is used as restoration filters. Wiener gives good result with less complexity but LR gives better PSNR than Wiener filter [15].

\subsection{Block Diagram}

Fig. 1 shows the block diagram of the our proposed method, in first step noise is added into image To remove motion blur from image, in second step image is restored using proposed restoration algorithms that is Wiener (filter 1) and LR (filter 2) as shown in fig 1. Finally both Images are fused using image fusion method to get the fused image [13].

\section{IMAGE FUSION}

In image fusion method the information from each of the given original images is fused together to form a resultant image whose quality is superior to the input images. DWT was performed on the original image then images are decomposed into four LL, LH, HL, and HH sub-bands [9, 10, 12]. By using the wavelet coefficient of source image it is possible to construct the fused wavelet coefficient map with the help of decision map.

The decision map shows each value which is the index of source image, some time it may be more informative on the corresponding wavelet coefficient. Then, we will actually make a decision on each coefficient. By using two type of fusion rule first pixel- based so only pixel values either max or average can consider and second is window-based so consider not only the corresponding coefficients, but also their close neighbors, say a $3 \times 3$ or $5 \times 5$ windows.

We used pixel level maxima rule in this work. On the basis of this fusion decision map of source images, we can make the wavelet coefficient map for fused image and then obtain the fusion image by inverse wavelet transform.

\section{EXPERIMENT RESULTS}

This paper shows the different size images such as $256 \times 256$ and $128 \times 128$ and then blurred those images.

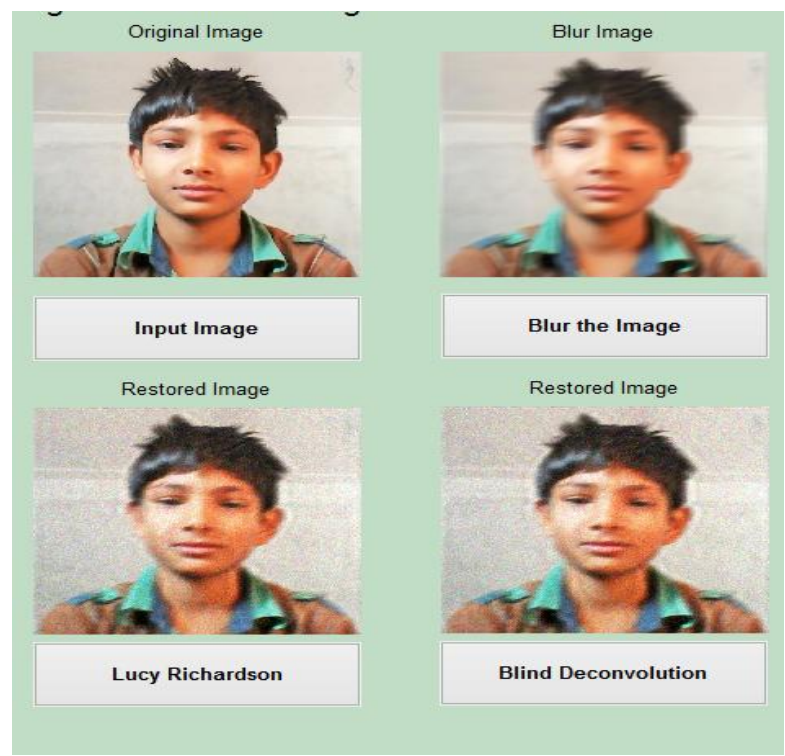

Fig 2: Original image of 256x246, blurred image and restored image by using Lucy Richardson and blind deconvolution

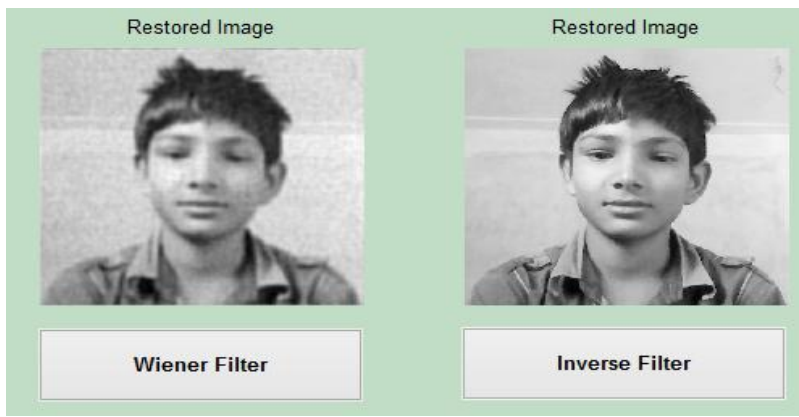

Fig 3 : Restored image by wiener Filter and Inverse filter for size of $256 \times 246$

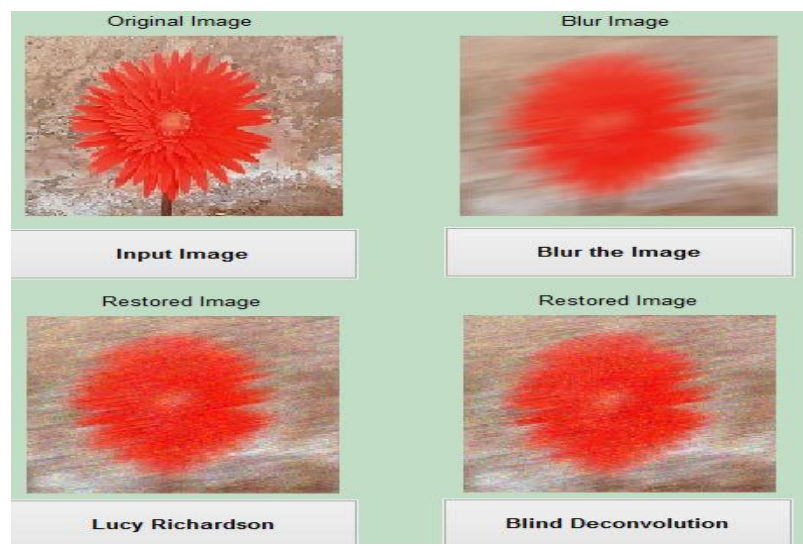




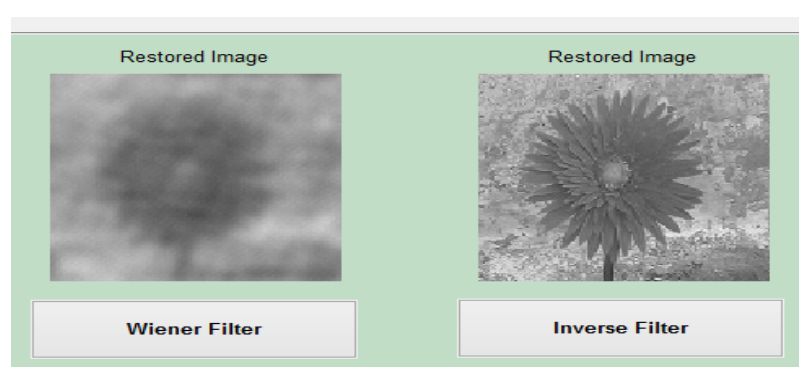

Fig 4 : Original image of size 128x128, blurred image, restored image by using Lucy Richardson, blind deconvolution by wiener Filter and Inverse filter.

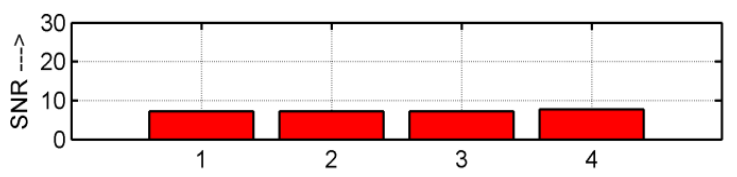

(a) Child Image

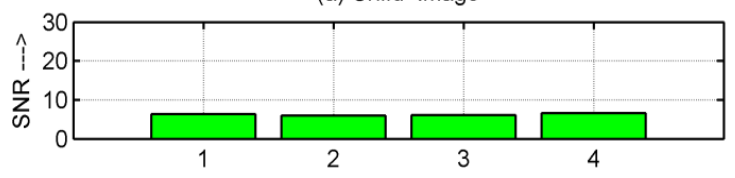

(b) Flower Image

1. Blurred Image 2. Fused Blind with Lucy Richardson 3. Fused Wiener with Lucy Richardson 4. Fused Lucy with regularised Filter

Fig 5: SNR for child image \& flower image

After that different restoration technique such as Lucy Richardson and blind deconvolution are applied. The figure 2 and figure 3 shows the original and resultant images of size 256X256. The figure 4 shows the original and resultant images of size $128 \times 128$. Then by using wiener filter and inverse filter to restore original image. By comparing obtained result it could be concluded that image recovered by inverse filter is comparatively better than other method. SNR and MSE parameter are calculated and shown in fig.5 and fig.6.
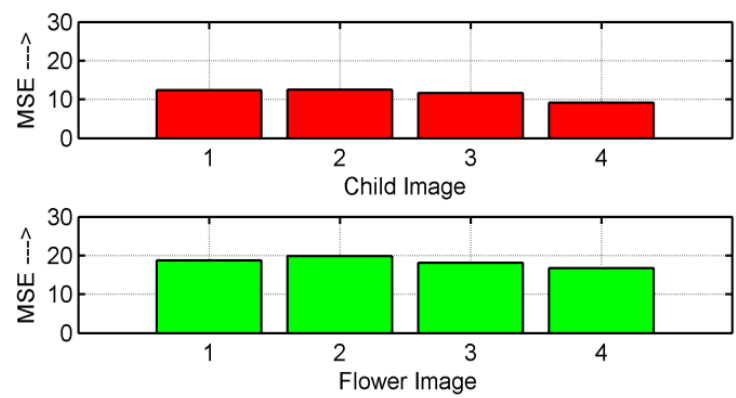

1. Blurred Image 2. Fused Blind with Lucy Richardson 3. Fused Wiener with Lucy Richardson 4. Fused Lucy with regularised Filter

Fig 6: MSE for child image \& flower image

\section{CONCLUSION}

In this work we implemented and compared the performance with filter such as Wiener filter, inverse filter Lucy Richardson and Blind de convolution. Results shows that Weiner filter with Image fusion reduce the computational complexity of image with better restoration results of image. It shows some experimental results of our work on wavelet based, proposed method and, together with, blind- de- convolution and wiener filter, Inverse filter methods. We can see that the results based on the suggested.

\section{REFERENCES}

[1] R. C. Gonzalez, R. E. Woods, S. L. Eddins, "Digital image processing using MATLAB" Pearson, 3rd Edition 2005

[2] A. U. Gupta, "Image Restoration Using Wavelet Based Image Fusion”, Vol. 3, 2 Feb 2011.

[3] A. K. Jain, "Fundamental of digital image processing", PHI 2005.

[4] Y. Xia, and M. S. Kamel "Novel cooperative neural fusion algorithms for image restoration, image fusion", Feb 2007.

[5] M.Tico,M.Vehvilainen,NokiaResearchCentreFinland,tico @ieee.org, "Estimation of motion blurs PSF from differently exposed Image frames."

[6] A. Levin, "Blind motion deblurring using image statistics", school of computer science and Engineering, The Hebrew University, MIT CSAIL, alevin@csail.mit.edu, Jerusalem.

[7] J. Portilla, V. Strela, M. J. Wainwright and E. P. Simoncelli, "Image Denoising Using Scale Mixtures of Gaussians in the Wavelet Domain", IEEE transactions on image processing, Vol. 12, No.11, Nov 2003.

[8] D. L. Hall and J. Llinas, "An introduction to multisensor data fusion" Processings of IEEE, Vol. 85, No. 1, Jan 1997.

[9] Deepak Kumar Sahu, M.P. Parsai, "Different image fusion techniques -a critical review", Vol. 2, Issue. 5, Sep.-Oct. 2012, Jabalpur MP, India

[10] Y. Kurmi and V. Chaurasia, "An image fusion approach based on adaptive fuzzy logic model with local level processing," Int. Jour. of Comp. Appl., Aug. 2015, vol. 124, no.1, pp. 39-42.

[11] D. Sharma, Y. Kurmi, and V. Chaurasia, "Formation of super- resolution image: a review," Int. Jour. of Emerging Tech. and Adv. Engg., Apr. 2014, vol. 4, no. 4, pp. 218-221.

[12] Y. Kurmi and V. Chaurasia, "Performance of haze removal filter for hazy and noisy images," Int. Jour. of Sci. Engg. and Tech., Apr. 2014, vol. 3 no. 4, pp. 437 439.

[13] S. Tiwari, K. Chauhan, and Y. Kurmi "Shadow detection and compensation in aerial images using MATLAB," Int. Jour. of Comp. Appl., June 2015, vol. 119, no.20, pp. 59.

[14] S. Sharma, S. Sharma and R. Mehra, "Image restoration using modified Lucy Richardson algorithm in the presence of Gaussian and motion blur" Volume 3, pp. 1063-1070, Number 8 (2013).

[15] Amit S. Ufade, B.K. Khadse, S. R. Sur Aikar, "Restoration of blur image using wavelet based image fusion", Vol.-2, no. 2, Dec, 2012. 